\title{
O osteocrânio de Proceratophrys boiei (Wied-Neuwied), P. appendiculata (Günther), P. melanopogon (Miranda-Ribeiro) e P. laticeps Izecksohn \& Peixoto (Anura, Leptodactylidae)
}

\author{
Eugenio Izecksohn ${ }^{1}$, Sérgio Potsch de Carvalho-e-Silva ${ }^{1} \&$ Isabela Deiss ${ }^{2}$ \\ ${ }^{1}$ Instituto de Biologia, Universidade Federal do Rio de Janeiro. Caixa Postal 68044, 21944-970 Rio de Janeiro, Rio de \\ Janeiro, Brasil. E-mail: eizecksohn@netyet.com.br; sergio@biologia.ufrj.br \\ 2 Programa de Pós-Graduação em Zoologia, Museu Nacional, Universidade Federal do Rio de Janeiro. Caixa Postal 68044, \\ 21944-970,Rio de Janeiro, Rio de Janeiro,Brasil.E-mail: ideiss@uol.com.br
}

\begin{abstract}
The osteocranium of Proceratophrys boiei (Wied-Neuwied), P. appendiculata (Günther), P. melanopogon (Miranda-Ribeiro), and P. Iaticeps Izecksohn \& Peixoto, (Anura, Leptodactylidae). Skull bones of four Proceratophrys Miranda-Ribeiro, 1920 species sharing long dermal appendages on eyelids - P. boiei (Wied-Neuwied, 1825), $P$. appendiculata (Günther, 1873), P. melanopogon (Miranda-Ribeiro, 1926), and P. Iaticeps Izecksohn \& Peixoto, 1981 - are herein studied. Proceratophrys boiei, P. appendiculata and P. melanopogon share higher lateral crests on the frontoparietals, quadratojugal corners similarly positioned in relation to the vertical level of the occipital condiles, cranial bones with granules and tubercles, and maxillaries with ventro-posterior pits, hence being considered members of a single group; P. laticeps, given the presence of temporal arcades (similar to Ceratophrys Wied-Neuwied, 1824), quadratojugal corners posterior to the vertical level of occipital condiles, wrinkled skull bones, and maxilaries without ventro-posterior pits, is nonetheless considered as a member of a distinct group.

KEY WORDS. Anatomy, frogs, skulls, Southeastern Brazil, taxonomy.
\end{abstract}

RESUMO. Foram comparados alguns ossos do crânio de quatro espécies de Proceratophrys Miranda-Ribeiro, 1920 que compartilham longos apêndices palpebrais, como sejam: P. boiei (Wied-Neuwied, 1825), P. appendiculata (Günther, 1873), P. melanopogon (Miranda-Ribeiro, 1926) e P. Iaticeps Izecksohn \& Peixoto, 1981. Com base na forma dos frontoparietais, (com cristas laterais elevadas), na posição dos cantos dos quadratojugais em relação ao plano vertical dos côndilos occipitais, na ornamentação das superfícies cranianas (com tubérculos e grânulos), e na presença de fossetas maxilares posteriores ventrais, $P$. boiei, $P$. appendiculata e $P$. melanopogon foram consideradas em um mesmo grupo. P. laticeps, entretanto, por apresentar arcadas temporais completas (semelhante a Ceratophrys Wied-Neuwied, 1824), cantos dos quadratojugais posteriores ao plano vertical dos côndilos occipitais, superfícies cranianas rugosas e ausência de fossetas maxilares posteriores ventrais, é considerada pertencente a um grupo separado.

PALAVRAS CHAVE. Anatomia, crânios, rãs, Sudeste do Brasil, taxonomia.

A partir da metade do século XX generalizou-se entre herpetólogos a tendência ao reconhecimento de grupos de espécies mais diversificados dentro dos respectivos gêneros, sem a proposição de novos táxons. Assim, LYNCH (1971) reconheceu em Proceratophrys Miranda-Ribeiro, 1920 dois distintos grupos: o grupo boiei [incluindo P. boiei (Wied-Neuwied, 1825), $P$. appendiculata (Günther, 1873) e $P$. fryi (Günther, 1873)], com as pálpebras providas de cornos alongados, e o grupo bigibbosa [incluindo P. bigibbosa (Peters, 1872) e P. cristiceps (Muller, 1884)] com as pálpebras possuindo apenas uns poucos tubérculos maiores. Izecksohn et al. (1998), estudando séries de exemplares de Proceratophrys que além dos apêndices palpebrais longos compartilham um apêndice rostral, reconheceram como válidas as espécies P. appendiculata, P. melanopogon (Miranda-Ribeiro, 1926), P. laticeps Izecksohn \& Peixoto, 1981 e P. moehringi Weigoldt \& Peixoto, 1985, e descreveram duas espécies novas: P. subguttata Izecksohn, Cruz \& Peixoto, 1998 e P. phyllostomus Izecksohn, Cruz \& Peixoto, 1998. Essas seis espécies diferem de $P$. boiei por essa última, além de não possuir apêndice rostral, apresentar cristas frontoparietais mais desenvolvidas. Com a intenção de reunir subsídios para uma melhor compreensão das afinidades entre essas espécies, são comparados aqui os osteocrânios de $P$. boiei, $P$. appendiculata, $P$. melanopogon e $P$. laticeps.

Revista Brasileira de Zoologia 22 (1): 225-229, março 2005 


\section{MATERIAL E MÉTODOS}

Foram preparados e examinados dois crânios de exemplares machos adultos de cada espécie estudada, obtidos nas mesmas localidades: Proceratophrys boiei do município do Rio de Janeiro [antigo Estado da Guanabara considerado por Bokermann (1966) como localidade tipo da espécie]; $P$, appendiculata de Teresópolis, Estado do Rio de Janeiro; $P$. melanopogon de Itatiaia, Estado do Rio de Janeiro; P. laticeps de Linhares, Estado do Espírito Santo (localidade tipo), todas localidades do Sudeste brasileiro. Os indivíduos de P. melanopogon, apesar de apresentarem comprimento rostro-cloacal reduzido para a espécie (ca. de $43 \mathrm{~mm}$ ), foram considerados adultos pois vocalizavam ao momento de sua captura. Os exemplares foram previamente fixados em formalina $10 \%$ e conservados em etanol $70^{\circ}$ GL. Para a clarificação, retirou-se a pele e usou-se $\mathrm{KOH}$, alizarina e glicerina. Para a confecção dos desenhos em vista dorsal, os crânios clarificados e desprovidos das mandíbulas foram inicialmente fotografados a prumo imersos em glicerina, apoiados no substrato pelos pré-maxilares e pelos quadratojugais. As figuras em vista ventral foram baseadas também em fotografias, mas foram corrigidas para se adaptarem aos contornos das vistas dorsais. Para as confecções dos desenhos em vistas lateral e occipital, os crânios foram limpos dos tecidos moles e fotografados a seco. Todo o material estudado pertence à Coleção de Anfíbios do Departamento de Zoologia da Universidade Federal do Rio de Janeiro (ZUFRJ).

\section{RESULTADOS}

Os crânios das espécies examinadas são compactos em decorrência dos ossos serem espessos, com articulações firmes, com flanges e ornamentações presentes. As ornamentações consistem de projeções como tubérculos, grânulos, rugas e cristas que podem estar presentes nas superfícies em contacto com a pele, criando aderências e exostoses. Essas se mostram principalmente sobre os frontoparietais, que possuem cristas laterais. Os frontoparietais se fundem, sem fontanelas. Os quadratojugais são completos e se articulam com o processo posterior dos maxilares completando o arco maxilar. Os nasais se articulam com os maxilares. O ramo zigomático do escamosal se articula com o maxilar.

O crânio de Proceratophrys boiei (Figs 1-4 e 17) se destaca pelo desenvolvimento das cristas frontoparietais, projetadas para cima e para os lados, e que após se dirigirem para trás com leve divergência, se dobram posteriormente em direção ao plano sagital, formando um ângulo quase reto. As cristas frontoparietais formam entre elas uma depressão acentuada. Tubérculos e grânulos estão presentes nos ramos zigomático e ótico dos escamosais, e nos nasais, onde formam crista. Alguns grânulos são visíveis sobre os frontoparietais. O ramo posterior de cada escamosal tem a forma de uma placa ampla quase horizontal que deixa, entre ela e o frontoparietal, um espaço igual ou mais estreito que sua largura. O plano vertical que passa pelo ângulo posterior dos quadratojugais corresponde aproximadamente ao que passa pelos côndilos occipitais. Uma pequena cavidade, aqui denominada fosseta maxilar, está presente na porção posterior da face ventral de cada maxilar (Fig. 2).

O crânio de Proceratophrys appendiculata (Figs 5-8 e 18) difere do de $P$. boiei por sua menor altura e pela ausência de grânulos e tubérculos, tendo cristas pouco acentuadas. Os nasais exibem crista lisa transversal. As cristas frontoparietais são pouco desenvolvidas, algo arqueadas, e é rasa a depressão entre elas. O ramo ótico de cada escamosal é estreito e deixa um espaço entre ele e a crista frontoparietal com cerca de três vezes a sua largura. As fossetas maxilares estão presentes (Fig. 6).

O crânio de Proceratophrys melanopogon (Figs 9-12 e 19) distingue-se do de $P$. boiei por possuir cristas frontoparietais menos desenvolvidas, algo convergentes para trás, ramos óticos dos escamosais estreitos deixando espaços entre eles e as cristas frontoparietais equivalentes a três vezes sua largura. O crânio de $P$. melanopogon difere do de $P$. appendiculata por sua maior altura, cristas frontoparietais mais desenvolvidas, com maior depressão entre elas, e pela presença de tubérculos e grânulos nos nasais e escamosais. Fossetas ventrais estão presentes na parte posterior dos maxilares, como nas duas espécies anteriores (Fig. 10).

Proceratophrys laticeps (Figs 13-16 e 20) possui um crânio cujo aspecto difere sensivelmente dos acima referidos, tanto por sua largura como pela forma triangular em vista dorsal. As cristas frontoparietais, presentes na metade anterior dos ossos, não se projetam para cima e a depressão entre elas é rasa. Os frontoparietais, em sua metade posterior, prolongam-se como placas até se articularem com placas dos escamosais, formando arcadas temporais não justapostas aos proóticos. A ornamentação das superfícies em contacto com a pele consiste de rugas rasas que se ramificam ou se anastomosam e não há grânulos ou tubérculos, exceto um par sobre os nasais. Os ângulos dos quadratojugais atingem um plano vertical bem posterior ao dos côndilos occipitais. Não há fossetas maxilares (Fig. 14).

\section{DISCUSSÃO}

Os frontoparietais mostram as principais diferenças entre os osteocrânios destas espécies. Os frontoparietais em Proceratophrys boiei, P. appendiculata e P. melanopogon mostramse mais ou menos escavados em conseqüência de suas margens serem expandidas para os lados e para cima, sendo que $P$. boiei mostra um maior desenvolvimento destas margens, que são mais espessas do que nas outras duas espécies. Os frontoparietais de $P$. laticeps diferem dos das demais espécies aqui estudadas por se apresentarem menos escavados medianamente, mostrando seus bordos laterais pouco expandidos na metade anterior e fortemente expandidos na metade posterior onde se prolongam horizontalmente até se articularem com os ramos temporais dos escamosais, que também são expandidos, formando arcadas temporais completas. As superfícies do crânio de $P$. laticeps que apresentam maior contato com a pele, como as dos frontoparietais, dos ramos zigomáticos dos escamosais, das porções faciais dos maxilares, e dos nasais, exibem rugas que se bifurcam e se anastomosam, sendo desprovidas de tubércu- 

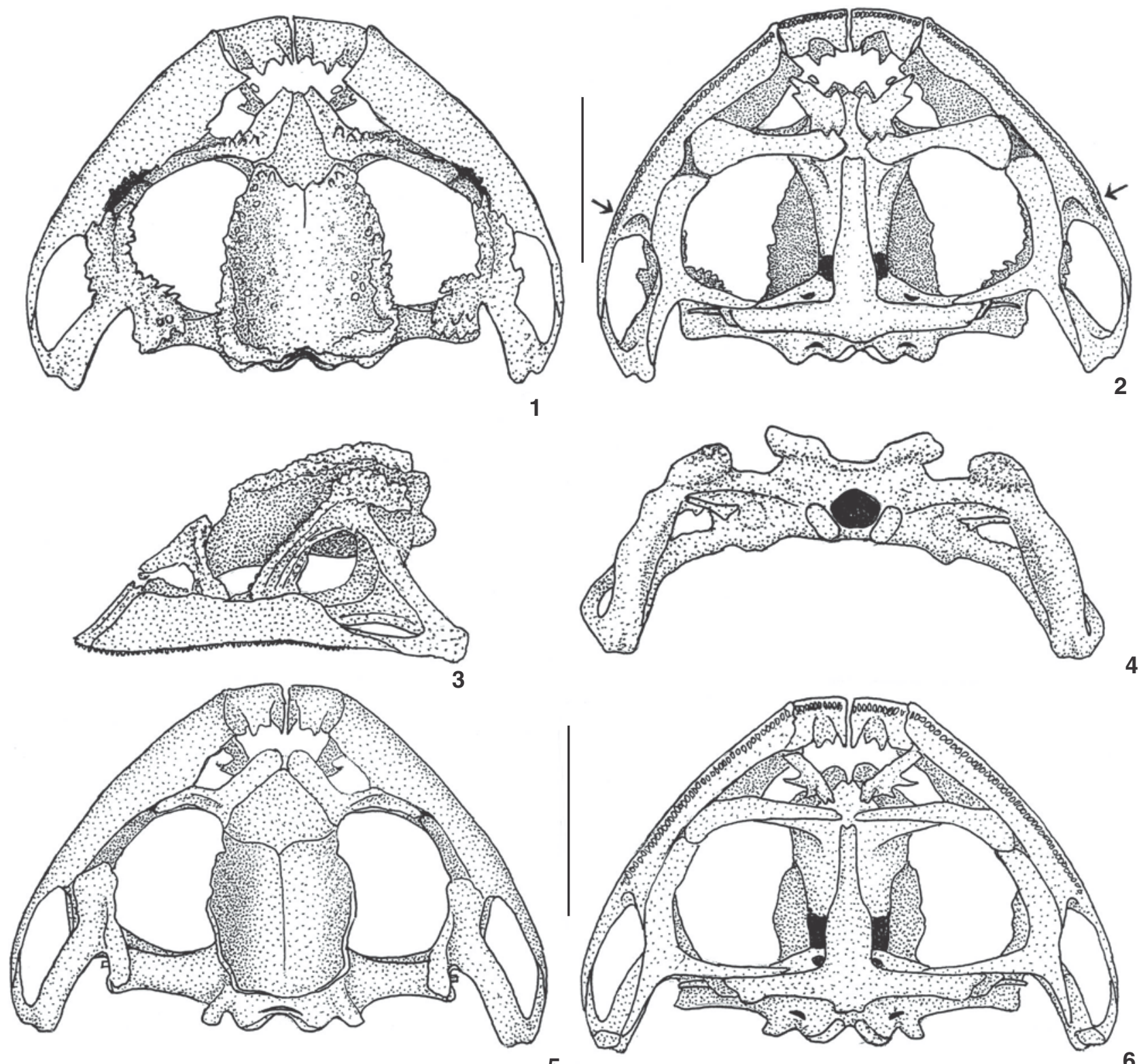

4
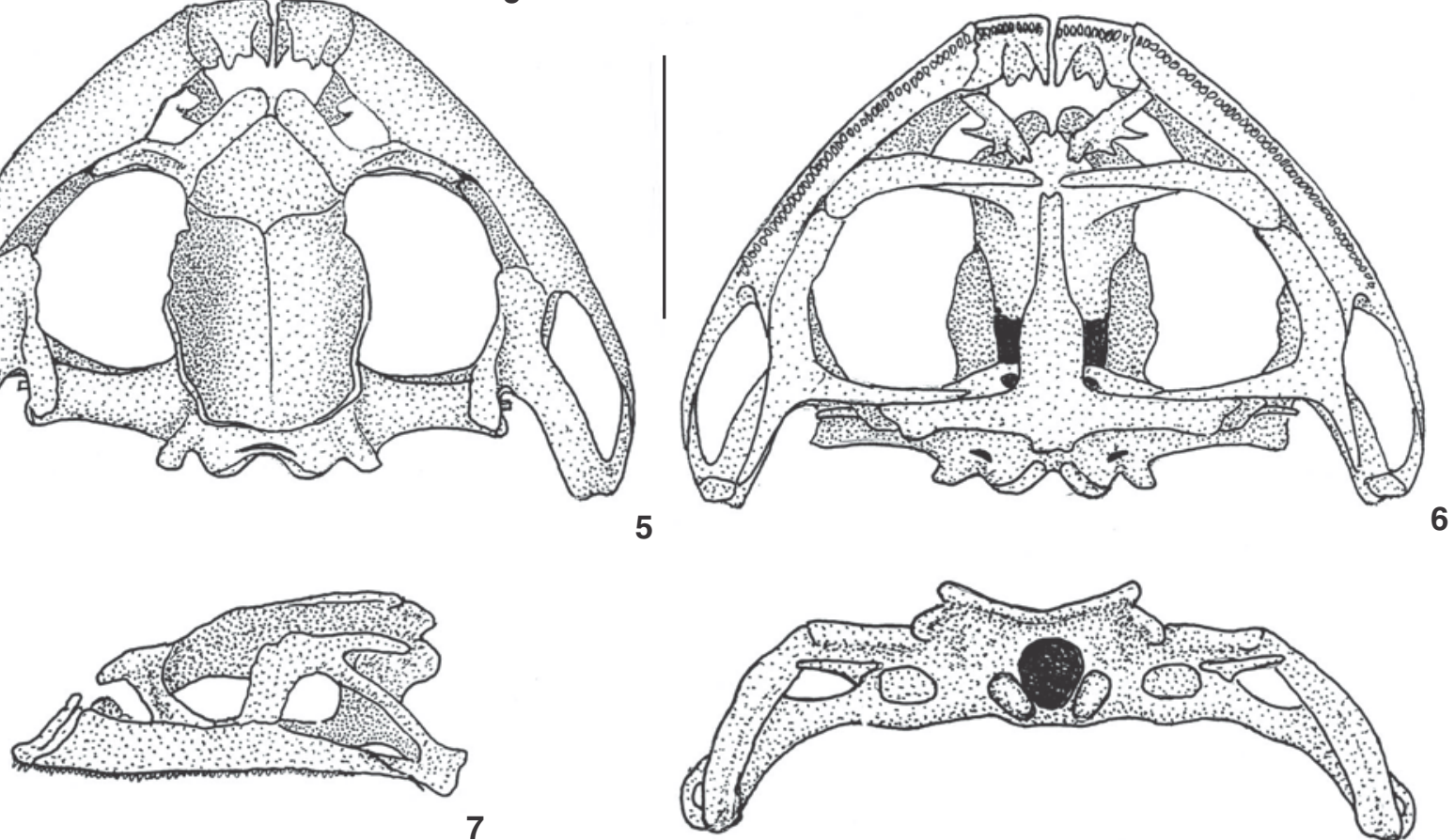

7

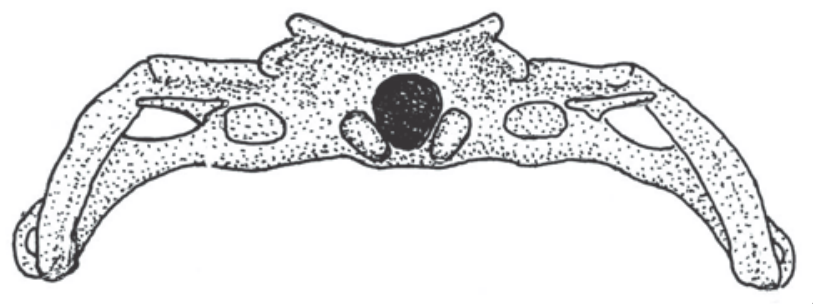

8

Figuras 1-8. Osteocrânio. (1-4) Proceratophrys boiei, ZUFRJ 638: (1) vista dorsal; (2) vista ventral (as setas assinalam as fossetas maxilares posteriores ventrais); (3) vista lateral; (4) vista occipital; (5-8) Proceratophrys appendiculata, ZUFRJ 9405: (5) vista dorsal; (6) vista ventral; (7) vista lateral; (8) vista occipital. Escala $=10 \mathrm{~mm}$. 

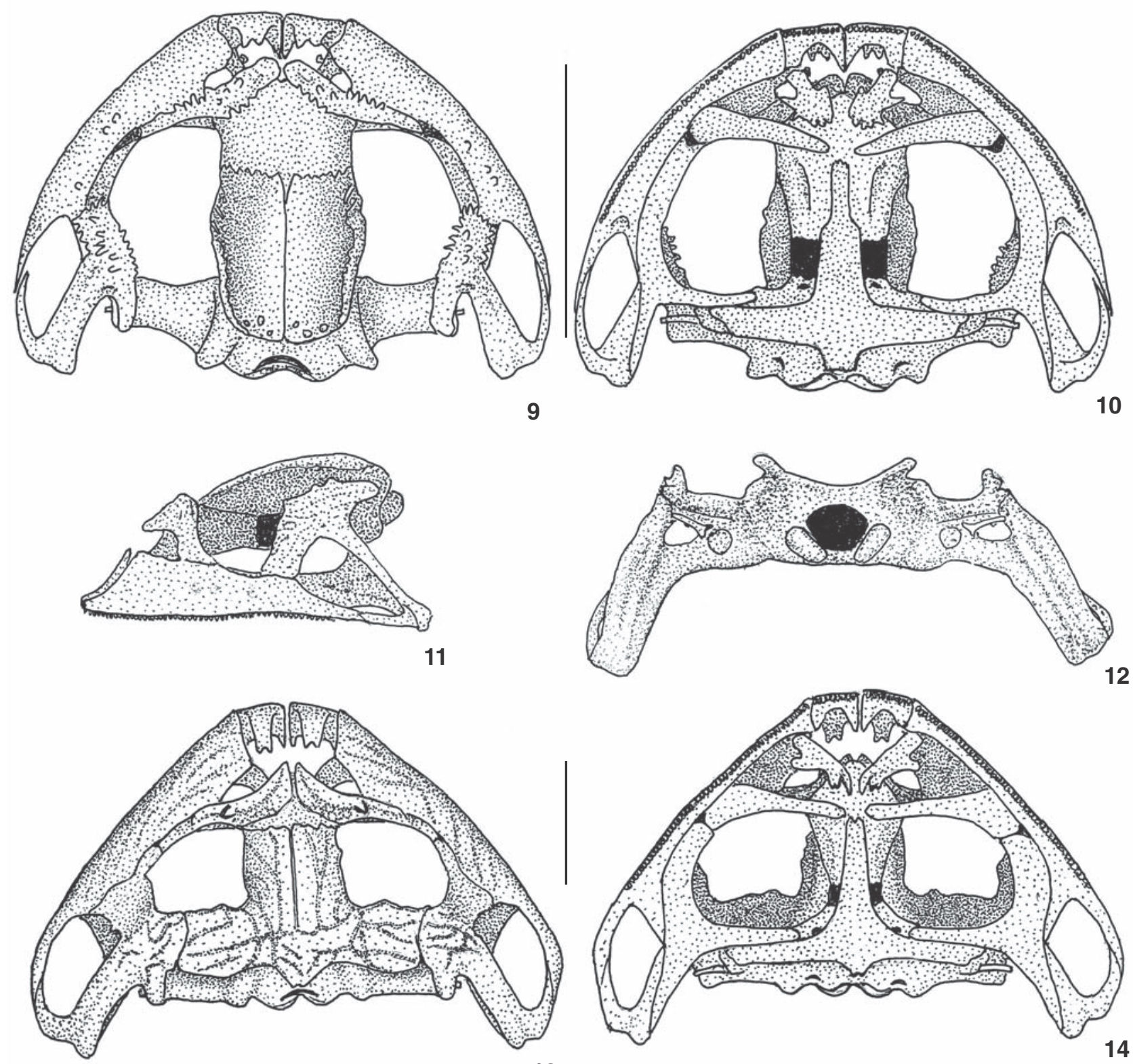

13
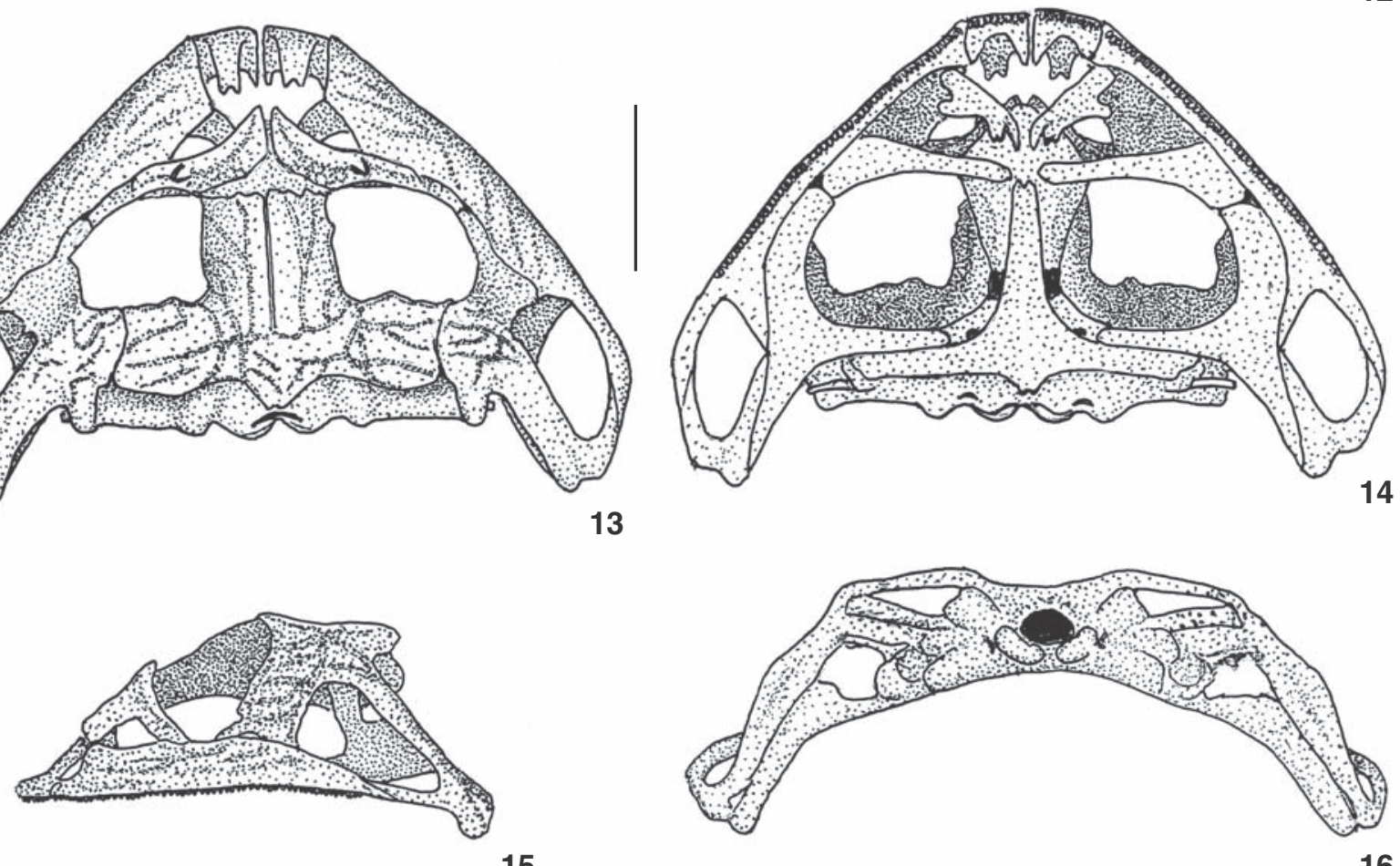

15

Figuras 9-16. Osteocrânio . (9-12) Proceratophrys melanopogon, ZUFRJ 8966: (9) vista dorsal; (10) vista ventral; (11) vista lateral; (12) vista occipital; (13-16) Proceratophrys laticeps, ZUFRJ 9379: (13) vista dorsal; (14) vista ventral; (15) vista lateral; (16) vista occipital. Escala $=10 \mathrm{~mm}$.

Revista Brasileira de Zoologia 22 (1): 225-229, março 2005 


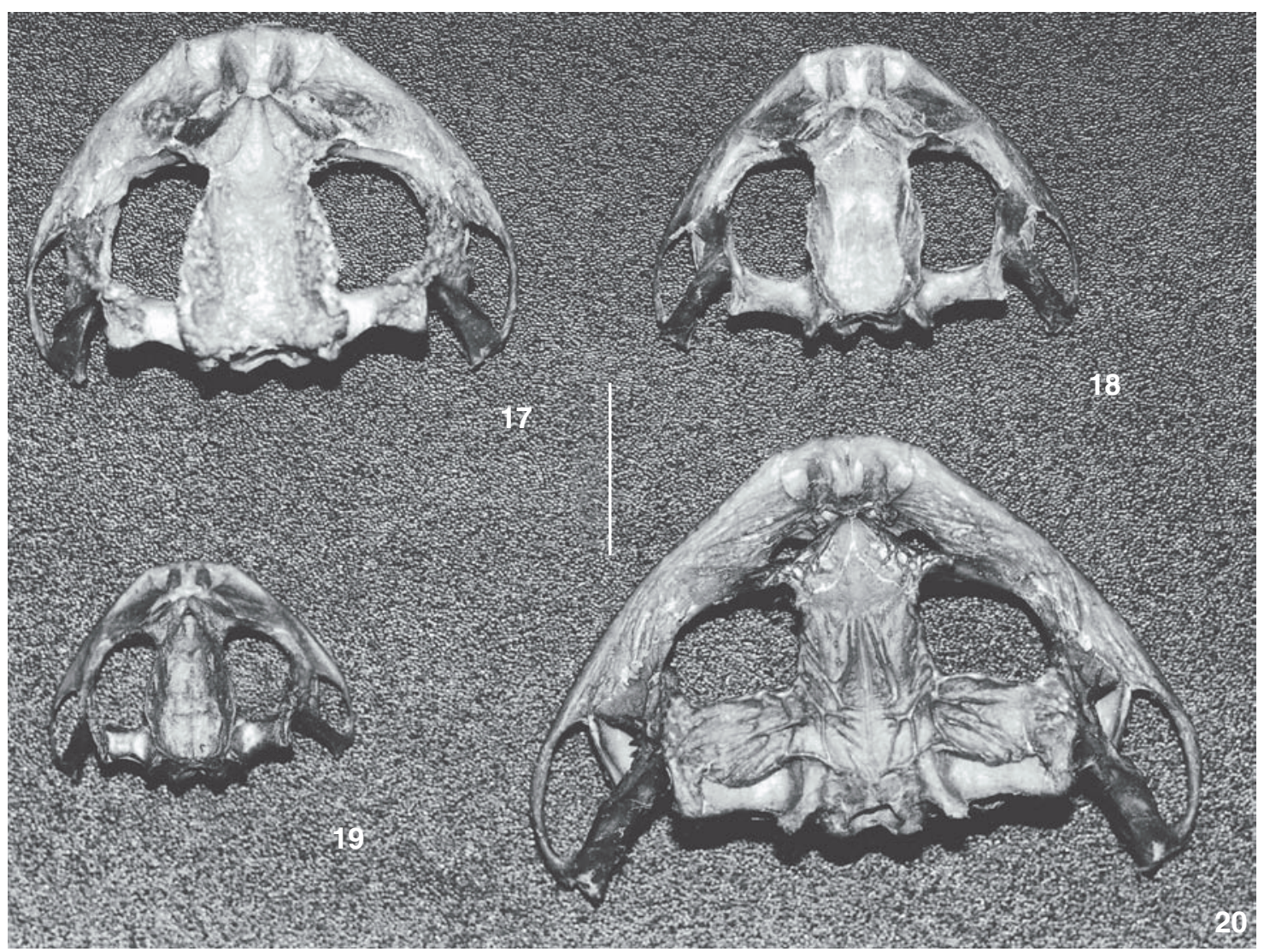

Figuras 17-20. Fotografia dos osteocrânios dos Proceratophrys examinados, em vistas dorsais: (17) P. boiei, ZUFRJ 638; (18) P. appendiculata, ZUFRJ 9405; (19) P. melanopogon, ZUFRJ 8966; (20) P. laticeps, ZUFRJ 9379. Escala $=10 \mathrm{~mm}$.

los, exceto um par nos nasais. As arcadas temporais de $P$. laticeps não são justapostas aos proóticos e formam o teto de fenestras temporais semelhantes às referidas por LYNCH (1971) para Ceratophrys Wied-Neuwied, 1824.

Apesar da falta de um apêndice cutâneo rostral em Proceratophrys boiei, e da presença de cristas frontoparietais mais desenvolvidas, são acentuadas as semelhanças do crânio desta espécie com os de $P$. appendiculata e de $P$. melanopogon, justificando sua reunião em um mesmo grupo, que provavelmente incluirá também outras espécies de Proceratophrys com apêndices palpebrais longos. Entretanto $P$. laticeps, por apresentar diferenças marcantes como a presença de arcadas temporais, um tipo peculiar de rugosidade na superfície dos ossos em contato com a pele, ângulos dos quadratojugais atingindo um plano transversal bem posterior ao dos côndilos occipitais, e por possuir maxilares sem fossetas ventrais posteriores, é aqui considerado como pertencente a um grupo separado.

\section{AGRADECIMENTOS}

Por haverem contribuído de diversas maneiras para que este trabalho fosse realizado, somos gratos a Oswaldo L. Peixo- to, Léo Nascimento, Renato M. de Jesus, Roberto V. Lima, Sérgio Maia, Bruno Filizola, Ana Maria P. Teles de Carvalho-e-Silva, Fanny Izecksohn e Ana Carolina de Queiroz Carnaval.

\section{REFERÊNCIAS BIBLIOGRÁFICAS}

BoKermanN, W.C.A. 1966. Lista anotada das localidades tipo de anfíbios brasileiros. São Paulo, EDUSP, 183p.

Izecksohn, E.; C.A.G. Cruz \& O.L. Peixoto. 1998. Sobre Proceratophrys appendiculata e algumas espécies afins (Amphibia, Anura, Leptodactylidae). Revista Universidade Rural, Série Ciências da Vida, Seropédica, 20 (1-2): 37-54.

LynCH, J. 1971. Evolutionary Relationships, Osteology, and Zoogeography of Leptodactyloid Frogs. Miscellaneous Publication, Museum of Natural History, University of Kansas, Lawrence, 53: 1-238.
Recebido em 01.VII.2004; aceito em 21.II.2005.

Revista Brasileira de Zoologia 22 (1): 225-229, março 2005 